\title{
4K Laboratory FOCAL
}

\author{
S. W. LINK \\ McMaster University, Hamilton, Ontario, Canada
}

\begin{abstract}
While FOCAL has become a commonplace calculational language in many small computer laboratories, the use of FOCAL as an experimental control system has been largely ignored. In the present paper, a $4 \mathrm{~K}$ FOCAL laboratory system is described. Extensions to the FOCAL function and command lists provide for integer manipulations, high- and low-speed output, and a variable $D O$ statement. By providing access to experimental peripheral devices, FOCAL can be used as a powerful operating system. As an illustration, a two-choice reaction time experiment is discussed, a FOCAL control program for this experiment is described, and an assembly language listing illustrates how changes to FOCAL can be made.
\end{abstract}

Like many of you, I began my computer training on a small computer. The selection of a small computer was not so much a matter of choice as of necessity, for at the time most computers were small-if not physically, then at least computationally. The Bendix G15-D was such a computer. The $\mathrm{D}$ signified the presence of a drum that revolved to expose, slowly, each of 1,024 words of bulk storage. The machine was user operated, and, because of modest operating tolerances, was abused by the vagaries of both humidity and temperature. We programmed it in hexadecimal, without an assembly language, but usually with what we conceived of as consummate skill and lofty logic. We soon learned the canard that while people are smarter than computers, computers are smarter than programmers.

As computers and their operating systems grew, so grew our nostalgia for the "hands on" feature of a small computer. We progressed to the 709 , the 7090 , the $\mathrm{B} 5500$, and a variety of CDC machines only to abandon them at the arrival of the first PDP-1. Our nostalgia abated, we advanced toward the use of small laboratory computers that were fast, simple to operate, reliable, but often contained only $4 \mathrm{~K}$ of core memory.

It was with this background that I approached reviewing Bernard Weiss' very fine new book, Digital Computers in the Behavioral Laboratory (1973) (Link, 1974). About the only criticism I had concerned the omission from the book of a chapter describing how FOCAL could be modified to provide a laboratory operating system. I claimed that FOCAL could be a powerful laboratory language; Bernard Weiss asked me to demonstrate how-so here we are.

My purpose, then, is to illustrate in rather simple terms how 4K FOCAL has been used in a laboratory where choice responses and their response times are always gathered and where the number of within-subject

The research described in this paper was supported by the National Research Council of Canada. The paper was aided by the support of Rockefeller University during my tenure as visiting Associate Professor of Psychology, Reprint requests should be sent to the Department of Psychology, McMaster University, Hamilton, Ontario, Canada. experimental outcomes is at most 30 -about the largest number that can be conveniently investigated during a 1 -h experimental session. The point of this example is to illustrate that a considerable savings in programming effort can be obtained by the modification of one manufacturer's user-oriented software.

The application of FOCAL to experimental control is not new. A number of previous papers have pointed to FOCAL applications, and some have even included illustrations of FOCAL programming together with patches designed to implement new instructions or permit input from external experimental devices (e.g., Reece, 1973; Siegel, 1972). At least one author has suggested, however, that FOCAL will require more than $4 \mathrm{~K}$ of storage for all but the simplest of experiments (Doll, 1972). Yet, for many laboratories, simple experiments are quite common, and the use of FOCAL will provide faster set-up time than could be obtained by assembly language programming or by the modification of some quite sophisticated laboratory packages (Matthews \& Wescourt, 1974; Millman, 1971).

The laboratory environment will determine how often, and how many additional, peripheral devices must be sampled or supplied with data by FOCAL. In this regard, our laboratory is similar to laboratories for which large operating systems have been written. Minor inspection would reveal the usual jungle of computer (PDP-8I), peripheral equipment, and assorted, if not tangled, cables connecting the computer to an experimental room. We run one subject at a time, in part because the stimulus equipment must sometimes be calibrated for each subject, but mostly because our experiments are lengthy within-subject psychophysical studies which do not require more than a single subject station. In addition to a high-speed reader/punch and ASR-33, we have a calligraphic display system (Griffin, 1968: Link. 1969), auditory and tactual stimulus control devices, indicat or lights, various types of response panels, a programmable clock, and a Tri-Data Cartrifile that has been taught to perform like a DEC disk monitor system. 


\section{A TWO CHOICE RT EXPERIMENTAL DESIGN WITH CUED PRESENTATION PROBABILITIES.}

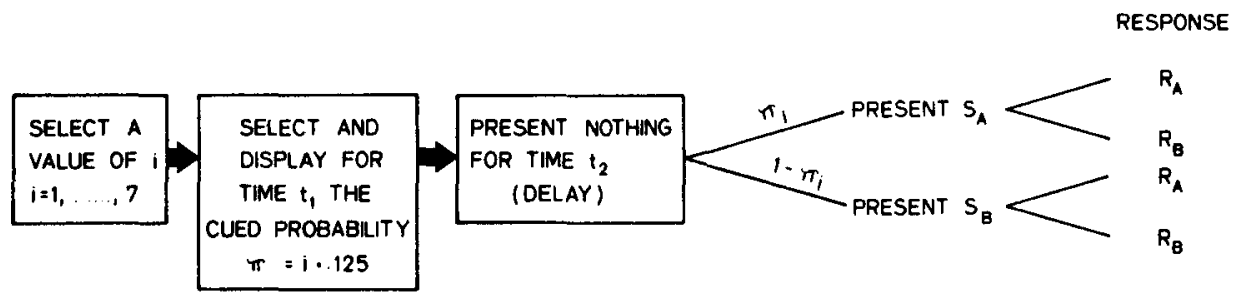

( 7 VALUES OF $\pi) \times(2$ STIMULU $) \times(2$ POSSIBLE RESPONSES $)=28$ POSSIBLE EVENTS PEF

Figure 1: A two-choice reaction time experimental design.

A typical laboratory experiment is illustrated in Figure 1. The purpose of this two-choice response time experiment was to bias a subject on a trial by trial basis toward one of two response alternatives, $R_{A}$ or $R_{B}$. A point of major theoretical interest was how correct and error response times would change as a function of response bias (Link, 1975).

The program controlling the experiment must perform the following tasks. At the start of each self-paced trial, the probability of presenting stimulus $\mathrm{S}_{\mathrm{A}}$, on the current trial, must be presented to the subject for a convenient length of time (T1). Thereafter, a blank display screen is presented (for time T2) to clear the visual field and provide a warning signal. Then stimulus $S_{A}$ is presented with a marginal probability equal to the displayed probability. The stimulus remains visible until the subject responds $R_{A}$ or $R_{B}$, and both the response made and the response time must be recorded and saved for later analysis. However, to provide the experimenter and the subject with a summary of results, we accumulated for each displayed probability value, $\pi_{i}(i=1, \ldots, 7)$, the numbers of responses of each type to each stimulus and the associated response times. Since there were seven values of $\pi$, two stimuli, two responses, and two response measures, the results from a single trial would occupy 2 of 56 data collection bins. At the end of the subject session, response frequencies and mean RTs for each stimulus-response combination and each value of $\pi$ were to be reported on the Teletype.

The first question we face in adapting FOCAL to our experimental needs is what changes to make. Any changes that are made must either replace or modify existing FOCAL routines or be patched to FOCAL in any unused space. It is, therefore, of primary importance that we have a clear idea of how free space can be obtained.

There are three methods of increasing the amount of available memory space. First, by deleting the extended functions (LOG, EXP, ATN, SINE, and COSINE), an area from $3206_{8}$ to $5400_{8}$ is made available. The size of this area is greater than the space occupied by Symbolic Editor. Second, by limiting the memory area used for storage of the FOCAL program, variables, and the push down list, we can reserve a memory area for patches or data storage. Last, there are many sections of 4K-FOCAL 69 that are unused, and some command routines, such as the $\mathrm{L}$ command, provide additional free space when deleted. By these simple methods, well over one-quarter of the $4 \mathrm{~K}$ memory is free to be used for storage of the FOCAL program, variables, push down list, and user defined routines.

Having determined the available free space, we now wish to use this space efficiently.

When a large number of variables are to be defined, and the values assumed by these variables are less than 4095 , an efficient use of free memory space can be made by treating these variables as integers. In contrast to variables defined by FOCAL, which require five contiguous memory locations, our integers each occupy only a single location. Naturally, deviating from the FOCAL definition of a variable will require additional programming space (approximately 18 locations), but this increase is offset by more efficient storage.

In the sample program below, integer manipulation is accomplished by defining two new FOCAL functions. The function FPUT $(X, Y)$ will convert to an integer $0<\mathrm{Y}<4095$ either the value $\mathrm{Y}$ or a fixed numerical value and place the integer value in Memory Location X (specified in decimal). An example of FPUT is shown in statement, 01.03 of the sample FOCAL program. We wished to fill Memory Locations 2342 to 2453 with zero. It can be seen that rather than using a FOCAL variable set equal to zero, a fixed integer value of 0 was used. To retrieve an integer already in memory, another FOCAL function FLST ( $x$ ) will convert the integer found in (decimal) Location $X$ to a FOCAL variable. For example, the command SZ=FLST(X) will set the 
FOCAL variable $\mathrm{Z}$ equal to the integer value found in Location $X$. These two FOCAL functions control input to and output from user selected areas of memory and vastly increase the capability of FOCAL to store experimentally obtained data values such as frequencies of stimulus-response pairings.

In addition to functions providing for integer storage and retrieval, other functions can increase the convenience of Laboratory FOCAL. To the version of FOCAL controlling the two-choice reaction time experiment, we have added a new random number generator and a routine to switch between high- and low-speed output. By setting $Z=F R A N()$, a pseudorandom number bounded by 0 and 1 will be generated. The random numbers so obtained have satisfied marginal probability, runs, and sequential tests for randomness. Switching between low- and high-speed output is accomplished by the instruction $S \mathrm{Z}=\mathrm{FSWP}($ ). Each execution of this instruction promotes a change from the current to the alternative output mode. High-speed output will, in many cases, obviate the need to devote large memory areas to storage of trial by trial results.

Other efficiencies are to be had by modifying or augmenting the FOCAL command list. In the present case, only two changes have been made. The first change is a modification of the Comment $(\mathrm{C})$ command. Normally, whenever a $\mathrm{C}$ is encountered, the FOCAL processor will simply ignore any subsequent characters up to the next text terminator. With a rather minor modification, the Comment command can also be made to clear all device flags. If peripheral devices are not to be serviced by a FOCAL interrupt handler, then it is particularly important that these devices and their flags be cleared at the beginning of a FOCAL program. Were these devices not cleared, FOCAL would sense an "illegal interrupt" and become quite confused.

The second command change provides for multiple branching beyond that offered by use of an IF statement. Suppose, for example, that on the basis of calculation from random numbers, experimental trial outcomes, or other methods, any 1 of 20 different resultant computations must be performed. A chain of IF statements would, of course, eventually lead to the desired computation. On the other hand, if the calculation yields a number that can be put into correspondence with numbers ranging from 1 to 20 , then a single branching statement could provide direct transfer to the desired computational sequence. To effect this operation, we have replaced the usual Library function, $\mathrm{L}$, with a routine which will transfer program control to an arbitrary FOCAL group number. Execution of the statement $\mathrm{L} \mathrm{X}$ will force a transfer of control to Group Number X. After execution of Group X, control is transferred back to the statement following LX. Thus, the multiple branching statement can be considered similar to a variable DO statement.

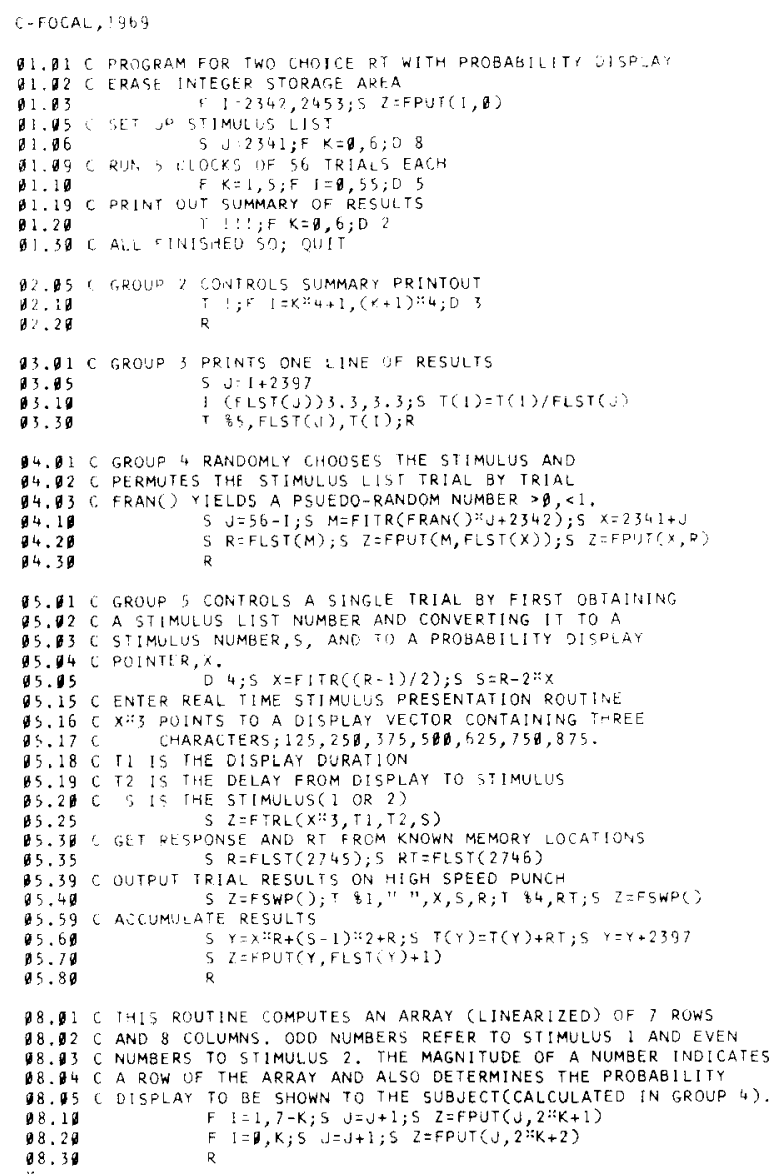

Figure 2: A 4K Laboratory FOCAL program for a two-choice reaction time experiment with cued presentation probabilities.

As an illustration of this command, let $\mathrm{Y}$ be a random number uniformly distributed between zero and one. If we want to execute 20 different, but equally likely, calculations based on the value of $Y$, we set $X=20^{*} Y+10$. The value of $X$ will range from 10 through 29 , and execution of the $\mathrm{LX}$ command will transfer control to Group Number X where the appropriate calculations can be performed.

A more powerful use of the $\mathrm{L}$ command is in sequencing program operation. The execution of the instruction $\mathrm{F} I=1, \mathrm{~N} ; \mathrm{L} X(\mathrm{I})$ provides an example. In this statement, the first group of statements to be executed is defined by $\mathrm{X}(1)$. A statement in Group $X(1)$ could modify any values in the vector $X(I)$. Furthermore, since the values of $\mathrm{I}$ and $\mathrm{N}$ can be changed by statements within Group $X(1)$, the sequence of program operation, after the completion of Group $X(1)$, can be altered. Since any group may operate on $\mathrm{I}, \mathrm{N}$, or any value of $\mathrm{X}(\mathrm{I})$, a program can be thought of as series of transitions from one group to another, where each group is sensitive to the current state of the program vector $X$. The use of the $\mathrm{L}$ command can provide great programming flexibility and can greatly simplify complicated multiple branching structures. 
The final problem to be faced in adapting FOCAL to the laboratory centers on access to experimental control devices. Access from the FOCAL control program can be provided by extension of the FOCAL function and command repertoire. At a minimum, a single function could pass to an assembly language subroutine all parameters required for execution of a single experimental trial while the subroutine would return to FOCAL the observed response measures. In this instance, the subroutine would assume complete control during an experimental epoch by disabling the FOCAL processor and operating in real time.

A second, and often preferable, method is to define a FOCAL function or command which will control a single experimental device such as a display device, a clock, and so forth. This method has the advantage of providing a single FOCAL operating system that can be used by experimenters not acquainted with assembly language programming. Although the implementation of this method may require substantial modification to the FOCAL interrupt handler, it provides a quite flexible experimental language (cf. Reece, 1973).

\section{A FOCAL EXAMPLE}

Figure 2 represents a FOCAL program which uses many of the function and command features described above. The program has been used to obtain two-choice RT data in an experiment designed to bias a subject toward one or the other of the two response alternatives. In this program, a single FOCAL function passes parameter values to an assembly language subroutine, FTRL, which assumes complete computer control during an experimental trial. Each group of statements is self-explanatory, given the numerous comments, but a short description of the main features of the program may be of some value.

Briefly, the FOCAL function FTRL (Y, T1, T2, S) was defined so that the arguments of the function could be computed in the body of the FOCAL program. These arguments refer to the presentation probability to be displayed to the subject, $Y$; the duration of that display, $\mathrm{T} 1$; the time interval between the termination of the probability display and the presentation of the stimulus, T2; and the value of the stimulus, S. Upon execution, FTRL controls all within-trial experimental events, waits for the subject's response, and then stores the response and response time in absolute memory locations. When FTRL has completed execution, the main body of the FOCAL program retrieves the response and response time. FTRL uses one page of memory.

The FOCAL program consists of two main sections. Group 1 is an executive routine that first clears all device flags and then initializes Locations 2342 to 2453 to zero. Then a 7 by 8 linearized array is filled with numbers which simultaneously indicate presentation probability and stimulus values. After these initial computations have been completed, five blocks of 56 experimental trials are run, and then summary results are printed on the Teletype.

Group 5 executes a single trial. First, a value is selected at random from the linearized array filled by Group 1. The value obtained is decomposed into a presentation probability indicator ranging from 1 to 7 and a stimulus value $S$. These values, together with $\mathrm{T} 1$ and $\mathrm{T} 2$, are passed to the assembly language trial controller by FTRL. After a response, control returns to FOCAL, and the values of the response and response time are obtained from Absolute Locations 2745 and 2746. All data summarizing the trial are then punched on paper tape, and summary statistics are gathered in two linearized arrays. At the end of Group 5, control is returned to Statement 1.10 .

What has been gained through this approach to the laboratory use of FOCAL is a bookkeeping system that relegates to FOCAL data handling operations. The control of a single trial is in the hands of FTRL where real time operation can be effected. Taking advantage of the great flexibility provided by FOCAL saves valuable programming time and yet allows for hands-on operation by laboratory personnel who are unfamiliar with assembly language programming. Furthe'rmore, debugging time is greatly reduced since the experimenter can simultaneously act as programmer and subject and can easily modify his FOCAL program to meet the demands of a new experimental design.

The major question most computer users ask concerning this application of FOCAL is how to get started. My experience is probably similar to that of others who have adapted FOCAL to their own purposes. First, one needs a model illustrating the assembly language programming required in defining new commands and functions. One of the best models is that of Reece (1973). However, to provide other illustrations, the appendix to this paper contains a PAL8 listing of the FOCAL modifications required to define FRAN, FLST, FPUT, FSWP, and changes to the $\mathrm{C}$ and $\mathrm{L}$ commands. In order to write a patch to FOCAL, one should have available a listing of FOCAL, the Advanced Focal manual (DEC-08-AJBB-DL, 1969). and the very useful monograph by Wrege entitled "FOCAL: How to write new subroutines and use internal routines." With these programming aids, an experimenter should have little difficulty in adapting FOCAL to his laboratory needs.

\section{APPENDIX}

The program listed below provides examples of patches to 4K-FOCAL 69. Some routines are identical to those employed by Reece (1973), and some other routines will be recognized by FOCAL users. 
PPATCH TO FOCAL FOR TWO CHOICE FAL 8-V7

IPATCH TO FOCAL FOR TWO CHOICE RT EXPERIMENT

JDEFINITJONS

Q153 INTEGER $=0153$

$136 \quad$ EFUN31 $=136$
456 SPNOR $=456$

4560 SPNOR $=456$
4542 PUSHA $=4542$

4543 PUSHF $=4543$

4540 PUSHJ $=4540$

1413 POPA $=1413$

$4544 \quad P O P F=4544$

$5541 \quad P O P J=554$

4557 RTL6=4557

$222 \quad \mathrm{PC}=22$

065 NAGSW $=65$
007 LINENO $=67$

0067 LINENO=

$0044 \quad$ EXP $=4$

1613 EVAL $=161$

1578 ATLIST $=1570$

374 FATABF $=37$

165 FNTABL $=2165$

163 COMGO $=1163$

$\begin{array}{ll}2600 & \text { SAVAC }=2600 \\ 3052 & \text { FRAN }=3052\end{array}$

ISPECIAL DEFINITIONS FOR PATCH

$A R G=J M S$ I $X A R G 1$

5200
6169 XPUT $=520160$

$583 \quad 5 W A P=7583$

$\begin{array}{ll} & \\ & \end{array}$

$354 \times$ XLST $=256$
$343 \times A R G=1343$

Q11 $C T=11$

B127 $M 2 P=127$

ICHANGES TO LOW CORE FOCAL

$\begin{array}{lllll}0001 & * 1 & & \\ 0001 & 6451 & & 6451 & \text { /CLEAR RESPONSE FLAG } \\ \text { OOAO2 } & 7000 & \text { NOP } & \end{array}$

QBOB3 $5404 \quad$ JMP 14

OQBO 42633 /FOCAL INTERRUPT HANDLER

$\$ 035: 35$

00354423 BOTTOM, LCOM-203 LAST FOCAL LOCATION

$170: 170$

90179 1343 XARG1, XARG

/LOCATION FILLED WITH FWA
/OF DISPLAY ROUTINE

D0172 5733 ADO, 5733 /ADOITION ROUTINE

BD173 4421 RANDOM, 4421 /CURRENT FLOATING

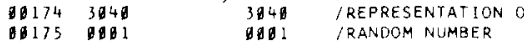

/CMANGES TO FUNCTION TABLES

93775200 KFNTABF+3 KTRL /MAIN TRIAL SEQUENCER

021792780 "FNTABL+3 $2700 \quad$ /COOE FOR FTRL $=4: T+2: R+$

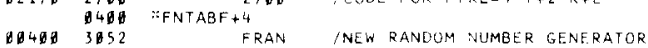

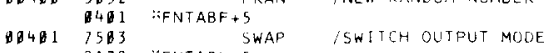

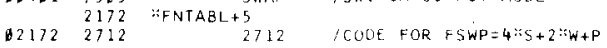

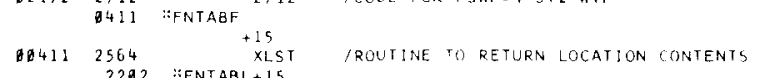

$022022652 \quad 2652$ /CODE FOR FLST $=4: 2+2:: 5+1$

DH4:12 616 "FNTABF+16 $\quad$ XPUT /RUUTINE TO PUT INTO CORE

D2203 2676 "FNTABL+16 2676 /CODE $=4 \because \mathrm{p}+2 * \mathrm{U}+\mathrm{T}$

$01574 \quad 1574$ "ATLIST+4 614 /REPLACE ADIR OF SYMBOL TABLE TYPEOUT

$01179 \begin{gathered}1170 \\ 4643\end{gathered}$

011734626 "COMGO+10 LCOM /THE L COMMANO NOW EXECUTES A DO $x$.

1217 7690 "1217 7500 TERASE COLONS

$6002: 6002$

/ROUTIN! TO GET AN ARGUMENT FROM A FUNCTION CALL.

01343 OAOH XXRG, OLL CLA IENTRY PUINT

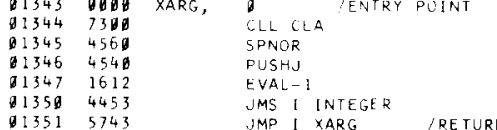

/ROUTINE TO GET CONIENTS OF A CORE ADDRESS.

$2564: \because \times L 5 T$

25644453 XLST, JMS I INTEGRR /GEI I

$02565 \quad 3377$

$2566 \quad 177$

$2567 \quad 711$

$\begin{array}{ll}2579 & 3045 \\ 2571 & 7919\end{array}$

$02572 \quad 3046$

a $2573 \quad 1376$

$02574 \quad 3044$

02576 0014

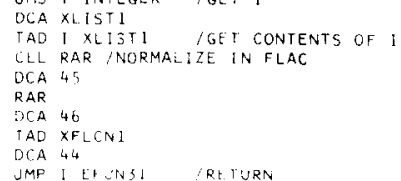

/ HANDOM NAMBEER GENERATOR: FRAN///

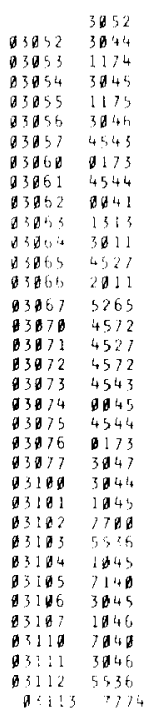

UCA 44

IAO RANOOM+1

DCA 46

PUSHF /FLAC $=R \div 2112$

RANDOM

POPF

EXX1+1 M / MULT BY 214

DCA CT

JMS I M2P

$152 \mathrm{CT} / \mathrm{MO} G E T R \because 2 \$ 16$

JMP $:-2$

JMS I ADD $\quad 1+R=R:(2 \uparrow 16+1)$

$\begin{array}{lll}\text { JMS I } & \text { M2P } & /: 2=R *(2117+2) \\ \text { JMS II } & \text { ADD } & 1+R=R *(2 \nmid 17+3)\end{array}$

EXP+1

RANDOM /NEW RANDOM NUMBER

DCA EXP+3 /CHOP TO 2 WQRDS

DCA EXP /MAKE A FRACIION

TAD EXP+1 /CHECK SIGN

$5 M A$ ELA

IMP I 136

TAB EXP+1

TRE TURN

CLL CMA

DCA $E X P+1$
TAD EXP+2

TAO

CMA $E$ CA $E$ O +

DCA EXP+2

TTAKE I'S COMPLEMENT

$-4$

/ALWAYS RETURN A POSITIVE NUMBER

ROUTINE TO EXECUTE a COMPUTED DO $X$ WITH $X$ AS

A VARIABLE. CALL AS $L x$ WHERE $x$ IS A PREVIOUSLY

DEFINED GROUP NUMBER. THIS ROU士INE REPLACES THE

IL COMMANO.

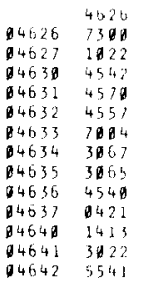

ROUTINE TO CLEAR ALL FLAGS AND BUfFERS. THIS

/ ROUTINE REPLACES THE C COMMAND IN FOCAL.

84643730 XCLR, CLL CLA

04644 611 642

04645 to2 012

84645020

04647 6 40

$0465 \quad 6132$

045516451

$44652 \quad 5356$

$04553 \quad 5456$

04055 tab 14

CLL CLA

TAD PC /5AVE FOCAL PROGRAM COUNTER

PUSHA ION THE PUSHDOWN LIST FOR RETURN

ARG /EVALUATE THE SYMBOL FOLLOWING

RAL

DCA LINENO

DCA NAGSW /SET ALL GROUP SWITCH

PUSHJ /EXECUTE THE GROUP

POPA /GET RETURN

POPA TGET RETURT

ROGRAM COUNTER

1032

192

CDISABLE CLOCK

HSSL ICLEAR AND SKIP ON RESP FLAG

6356 FREEZE SCOPD

6456 TCLEAR OUTPUT BUFFER

JMP I X:LRI IRETURN

LXPERIMENTAL ROUTINE FOR TWO CHOICE R

CALL WITH S ZFFTRL $(X: 3, T 1, T 2,5)$

$x=9, \ldots, 6$ SPECIFIES THE TRIAL TYPE, $: 3$ GIVES TABLE LOCATION

T: = OURATION OF PROBABILITY DISPLAY IN . I SEC. UNITS

$Z=$ INTERYAL BETWELN PRQB. DISPLAY ANO STIM.C.I SEC. UNITS

I H COOING OF :HIS ROUIINE WILL HE UNIQUE TO INDIVIOUAL

ABQRATORIES AND HAS BEEN OMITTED FROM THIS LISTING.

FOR THE PURPOSE OF T:IIS LISTING THE AVAILABZE SPACE

RUUTINE TO PUT DATA INTU CORD LOCATION.

ALL AS $5 Z Z=F$ = UTS $X$, Y $)$, WHERE $X$ IS ADDRES

IN DECIMALS ANO

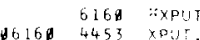

16160 4453 XPUT, JMS I INTEGER /GET $X$

06161336

061624570

$06163 \quad 376$

861643536

DCA XPUTl

DCA I XPUT? LATA VALUE

XPUT,

M IRETURN FROM CAL

RUIIIINE TO CHANGE OUTPUT MODE FROM LOW TO HIGH OR FROM

HIGS TO IOW SPEED. EACH CALL. REVERSE 5 THE MODE.

07503 $101 \mathrm{t}$ 5WATH, TAD 16 /WAIT fOR OUTPUT TO FINISH

07504764 B 57 CLA

JMP -2

TAD CURDEV

(CURDEV $)=20$ OR -20

UCA CURUEV

TAD ADDRS

DCA 11

TAD I I

SNA

SNAP I EFUN3I IRETURN

OCA PLACE

TAO I PLACE

TAD CUROEV

$\begin{array}{lll}07320 & 1324 & \text { TAD CUROEV } \\ 7521 & 37 \% 3 & \text { DCA I PLAC } \\ 7522 & 5313 & \text { JMP LOOP }\end{array}$

$\begin{array}{lll}97328 & 1324 & \text { TAD CUROE } \\ \text { O } 7521 & 3733 & \text { DCA I PLACE } \\ 7522 & 5313 & \text { JMP LOOP }\end{array}$

OTS23 BOH P'LACE,

O524 20 CURUEV, 2A ADORS /SET INITIALLY FOR LOW TO HIGH SPEED 


\section{LINK}

$\begin{array}{llll}07526 & 2606 & 2606 & 16041 \\ 07527 & 2610 & 2610 & 15042 \\ 07530 & 2615 & 2615 & 76044 \\ 7531 & 2711 & 2711 & 16046 \\ 0532 & 2762 & 2762 & 16046 \\ 7533 & 080 & 000 & \end{array}$

YFLCN 257

XLIST! 25 ?

$\begin{array}{ll}\text { XLST } & 2564 \\ \text { XPUT } & 5160\end{array}$

$\begin{array}{ll}\text { XPUT } & 5160 \\ \text { XPUT } & 6165\end{array}$

XTRL 5200

ADD $\quad 172$

ADDRS 7525

ARG 4571

BOTTOM IQBS

COMGO 1163

$C T$ O11

CURDEV 7524

DFUN3I 136

EVAL 1613

EXP \$4

FNTABF 1374

FNTABL 2165

FRAN 3052

INTEGE OOS

LCOM 4626

LOOP 7513

$\begin{array}{ll}M 2 P & 127 \\ M 4 & 3113\end{array}$

NAGSW DG5

PC 1022

PLACE 7523

POPA 1413

POPF 4544

POPJ 5541

PUSHA 4542

PUSHF 4543

PUSHJ 4540

RANDOM 173

RTL6 4557

SAVAC 2600

SHOW \#171

SPNOR 4560

SWAP 7503

SWITCH 7506

XARG 1343

XARGL

XCLR: 4655

\section{REFERENCES}

Doll, T. J. A 4-K computer language for experimentation with human subjects. Behavioral Research Methods Instrumentation. 1972, 4, 27-31.

Griffin, J. D. A. Investigation of CRT control room displays using a computer. Second Canadian Symposium Proceedings, DECUS, Toronto, 1968.

Link, S. W. A computer controlled laboratory for visual perception and human learning. Third Canadian Symposium Proceedings, DECUS, Toronto, 1969.

Link, S. W. Deus Ex Machina. Contemporary Psychology, 1974 $19,8,596-597$

Link, S. W. The relative judgment theory of two choice response time. Journal of Mathematical Psychology. 1975, 12.

Matthews, P., \& Wescourt, K. Imlac control program for psychological experiments. Department of Psychology. Stanford uiversity, Stanford, California, 1974

Millman, B. PSYPAL: A computer language for the control of psychological experiments. Department of Psychology Technical Report, University of Calgary, Alberta, 1971.

Reece, $\mathrm{P}$. Some simple I/0 patches for $4 \dot{\mathrm{K}}$ FOCAL. Decuscope $1973,12,23-29$.

Siegel, W. Combining FOCAL and assembly language. Behavioral Research Methods \& Instrumentation. 1972, 4, 105-106.

Weiss, B. Digital computers in the behavioral laboratory. New York: Appleton-Century-Crofts, 1973

Wrege, D. FOCAL: How to write new subroutines and use internal routines. DECUS: FOCAL-17.

Advanced FOCAL technical specifications. Maynard, Mass: Digital Equipment Corporation, DEC-08-AJBB-DL, 1969. 